\title{
Birefringence Caused by the Presence of Permanent Dipoles and Its Possible Threat on the Accuracy of Traditional Surface Plasmon
}

\author{
Po-Yu Tsai ${ }^{1}$, Chien-Jung Liao ${ }^{2}$, Wen-Kai Kuo ${ }^{1}$, Chungpin Liao ${ }^{1,2,3}$ \\ ${ }^{1}$ Graduate School of Electro-Optic and Materials Science, National Formosa University (NFU), Huwei, Yunlin, Taiwan, China; \\ ${ }^{2}$ Advanced Research and Business Laboratory (ARBL), Taichung, Taiwan, China; ${ }^{3}$ Chakra Energetics, Inc., Taipei, Taiwan, China. \\ Email: \{marveltsai, lchienjung\}@gmail.com, wkkuo@nfu.edu.tw, cpliao@alum.mit.edu
}

Received January $6^{\text {th }}, 2011$; revised January $28^{\text {th }}, 2011$; accepted February $1^{\text {st }}, 2011$.

\begin{abstract}
In arriving at the more intuitive "scattering form" of the Fresnel equations, microscopic physical electric and magnetic dipoles were rigorously employed as the source of electromagnetic waves by Doyle et al. Motivated by such an approach, the authors started to speculate how the incorporation of permanent dipoles might affect Brewster angle of a specific optical material. It is found that in the presence of permanent dipoles, not only is the Brewster angle dependent on the incident light power as well as the dipole orientation, but also that two conjugate incident light paths result in distinctively different refractions. Experiments on dipole-engineered polyvinylidene fluoride films show that by way of adding/reducing permanent dipole density and varying orientations, the aforementioned theoretical predictions can be evidenced unambiguously in the visible light range. Further, effective polarization density can be quantified from the above experiments subjected to different dipole engineering processes. As a result, the traditionally elliptic contour of a slanted two dimensional section of the refractive index ellipsoid now manifests symmetric open splittings at near the traditional incident angle. It implies that severe challenge to the accuracy of traditional surface plasmon resonance measurements may arise in the presence of permanent dipoles of various morphologies, such as in the forms of nano-particles or membrane double layers.
\end{abstract}

Keywords: Surface Plasmon Resonance, Fresnel Equations, Brewster Angle

\section{Introduction}

Traditionally, lightwave incidence, reflection, and refraction at interfaces among varying materials are well described by the widely-known classical Fresnel equations [1]. This set of equations even quantifies the power distribution and phase lags, among refracted light beams [1]. However, it fails to provide a clear, yet rigorous, picture as to how the real physical causes behind all these macroscopic optical phenomena - i.e., the microscopic electric and magnetic dipoles-work to come up with such macroscopic results. Lately, having being motivated by such microscopic insight (i.e., quantum scattering) and derivation of the "scattering" form of Fresnel equations [2], two of the current authors proposed a theory to alter Brewster angle of a specific material by incorporating permanent dipoles externally in a predetermined manner [3]. Among its predictions, the traditionally fixed Brewster angle of a specific material now not only becomes dependent on the density and orientation of incorporated permanent dipoles, but also on the incident light intensity (more precisely, the incident wave electric field strength). Further, two conjugated incident light paths would give rise to different refracted wave powers [3].

To quantitatively verify such theoretical predictions, this current work performs experiments on various dipole-engineered PVDF (polyvinylkidene fluoride) films in the visible light range. Then, based on the secured experimental data, the effective polarization number densities of permanent dipoles within these PVDFs are extracted for the plotting of refractive index ellipses (i.e., general slanted sections of the refractive index ellipsoids) to compare with traditional ones. It is revealed that these ellipses manifest open splits at large incident angles. Further, as a result of this finding, the authors point out the possibility of severe errors in traditional SPR (surface plasmon resonance) measurement when in the presence of permanent dipoles. 


\section{Alteration of Brewster Angles}

In arriving at the "scattering" format Fresnel equations, Doyle et al. [2] rigorously expressed the incident light electric field of a p-wave (i.e., a TEM wave with its electric filed on the plane-of-incidence (POI)) as a functional of the induced electric $(P)$ and magnetic $(M)$ dipole polarizations (i.e., dipoles per unit volume) as $[2,3]$ :

$E_{i}^{p}=\left[-\frac{P}{\varepsilon 0} \cos \left(\theta_{t}-\theta_{i}\right)-\frac{M \sqrt{\mu 0}}{\sqrt{\varepsilon 0}}\right] \times\left[\frac{\sin \theta_{t}}{2 \cos \theta_{i} \sin \left(\theta_{t}-\theta_{i}\right)}\right]$

It is by way of the above $\cos \left(\theta_{t}-\theta_{i}\right)$ relation between the induced electric dipole polarization $P_{\text {induced }}$ and the incident electric field $E_{i}^{p}$ that the authors figured out how an incorporated permanent polarization can enter into this light scattering picture (see Figure 1 (a)), with $\theta_{i}$ and $\omega$ being the incident angle and angular frequency of the incident light). Namely, with extra embedded permanent polarization $P_{0}(\omega)$ within a nonmagnetic material ( $M=0$ ) host (see, Figure 1 (a)), the total electric dipole contribution to $E_{i}^{p}$ becomes:

$$
\begin{gathered}
P_{\text {induced }} \cos \left(\theta_{t}-\theta_{i}\right)+P_{0} \cos \left(\theta_{t}+\theta_{i}\right) \text { i.e., } \\
\varepsilon_{0}\left(\varepsilon_{r t}-1\right) E_{t}^{p} \cos \left(\theta_{t}-\theta_{i}\right)+P_{0} \cos \left(\theta_{0}+\theta_{i}\right)
\end{gathered}
$$

or

$$
\varepsilon_{0}\left(\widetilde{\varepsilon}_{r t}-1\right) E_{t}^{p} \cos \left(\theta_{t}-\theta_{i}\right)
$$

we thus have [3]:

$$
\tilde{\varepsilon}_{r t}=\frac{P_{0}}{\varepsilon_{0} E_{t}^{p}} \frac{\cos \left(\theta_{i}+\theta_{0}\right)}{\cos \left(\theta_{t}-\theta_{i}\right)}+\varepsilon_{r t}
$$

where in $\theta_{0}$ denotes the declination angle of the electric polarization with respect to the interface (see, Figure 1).

If, instead of picking the incidence from the left hand side as depicted in Figure 1 (a), a conjugate path, i.e., from the right hand side, is taken (see, Figure 1 (b)), then the formula for (2) becomes [3]:

$$
\tilde{\varepsilon}_{r t}=\frac{P_{0}}{\varepsilon_{0} E_{t}^{p}} \frac{\cos \left(\theta_{i}-\theta_{0}\right)}{\cos \left(\theta_{t}-\theta_{i}\right)}+\varepsilon_{r t}
$$

Unconventional Brewster angle can be found by putting (2) and (3) into the p-wave Brewster equation [1,3]:

$$
\tan ^{2} \theta_{B}^{P}=\frac{\varepsilon_{r t}\left(\varepsilon_{r t}-\mu_{r t}\right)}{\varepsilon_{r t} \mu_{r t}-1}
$$

where the subscripts " $i$ " and " $t$ " denote the incident and transmitted sides of the interface, respectively, and $\theta_{B}$ represents the Brewster angle.

In other words, the traditionally fixed Brewster angle of a specific material now not only becomes dependent

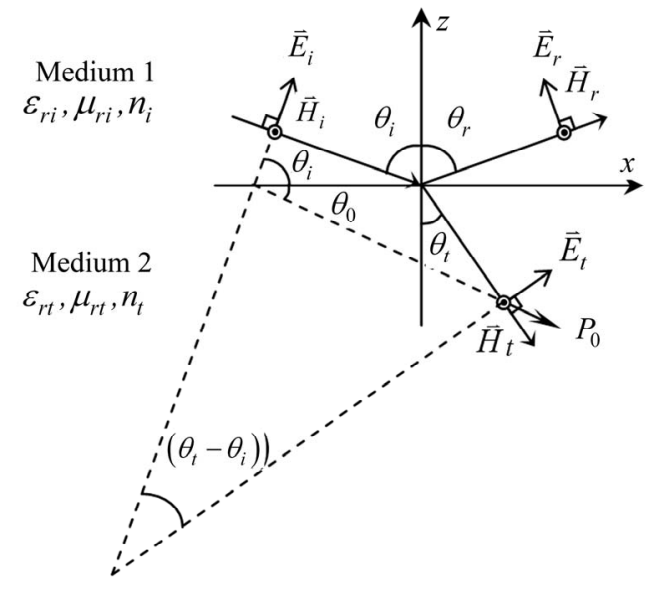

(a)

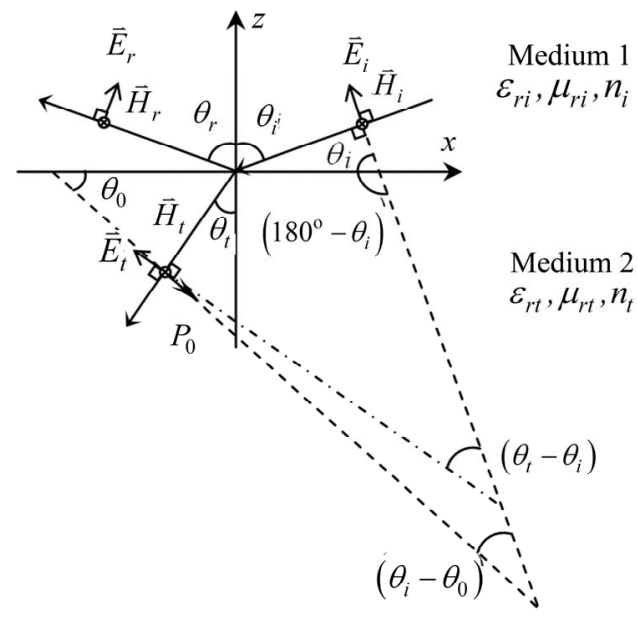

(b)

Figure 1. p-wave configuration at the interface and the orientation of embedded permanent electric dipoles (courtesy of Doyle [3]).

on the density and orientation of incorporated permanent dipoles, but also on the incident light intensity (more precisely, the incident wave electric field strength). Further, two conjugated incident light paths would give rise to different refracted wave powers $[3,4]$.

As a side remark, the reason s-wave (with its electric field perpendicular to the plane-of-incidence) is not considered here is due to the fact that it does not encounter the Brewster angle in the light frequency range normally (see, e.g., [1]).

\section{Experiments}

\subsection{Characteristics of Processed Polyvinylidene Difluoride}

With a bonding pattern of $-\left(\mathrm{CH}_{2}-\mathrm{CF}_{2}\right)_{\mathrm{n}}-$, PVDF (polyvinylidene fluoride) is a nonmagnetic, positive uniaxial ferroelectric polymer of birefringence of $n_{e}=1.4246$ 
and $n_{o}=1.4183$, respectively [5,6]. Up to this day, its various known morphologies of crystallization include: $\alpha$ (TGTG') , $\beta$ (TTTT) $[7,8], \gamma$ (TGTG or TG'TG') and $\delta$ (TTTG or TTTG') phases [7]. Among them, the $\alpha$ phase is essentially non-polar from which a $\beta$ phase film can be obtained from mechanically stretching the $\alpha$ phase. Another poled- $\beta$ phase is secured when further subjecting the previous $\beta$ phase to strong contact electric poling of more than $200 \mathrm{kV} / \mathrm{cm}$ [8]. Hence, both the $\beta$ and poled- $\beta$ phase molecules demonstrate much greater ferroelectricity and piezoelectricity even though orientations of their permanent dipole molecules are still far from being aligned along the imposed stress and electric field.

\subsection{Experiment on Varying Brewster Angle}

The experimental set up is as arranged in Figure 2, where a light beam of $0.686 \mathrm{~mm}$ radius from the $\mathrm{He}-\mathrm{Ne}$ laser (of the wavelength of $632.8 \mathrm{~nm}$ ) is converted into p-wave mode after getting through the polarizer. Two double convex lenses, with focal lengths being $12.5 \mathrm{~cm}$ and $7.5 \mathrm{~cm}$, respectively, are for shrinking down the beam radius to $0.19 \mathrm{~mm}$ to reduce the width of light reflection off the PVDF surface. The reflected light is then further focused by a lens (of $2.54 \mathrm{~cm}$ focal length) before reaching the diode power detector. An incident angle range is scanned from $50.5^{\circ}$ to $59.5^{\circ}\left(\theta_{i}\right)$ with an accuracy of $0.015^{\circ}$, and then its conjugate range from $-50.5^{\circ}$ to $-59.5^{\circ}\left(-\theta_{i}\right)$, while the incident light intensity is varied between $100 \%(8.54 \mathrm{~mW})$ and $10 \%$ power by moving an attenuator into or withdrawing from the beam path.

\section{Results and Analyses}

\subsection{Experiment Results}

The fitted curves for the measured Brewster angles for the two conjugate incident paths, under $100 \%$ and $10 \%$ laser beam intensities, on both the $\beta$ - and poled- $\beta$ films, are shown in Figure 3 and Figure 4, respectively. It can be seen that Brewster angles measured via the two conjugate incident paths differ considerably. Such difference becomes more outstanding on the poled- $\beta$ PVDF film,

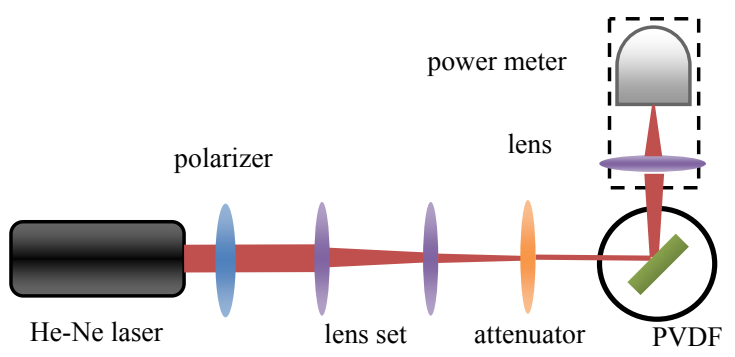

Figure 2. Configuration of Brewster angle measurement.

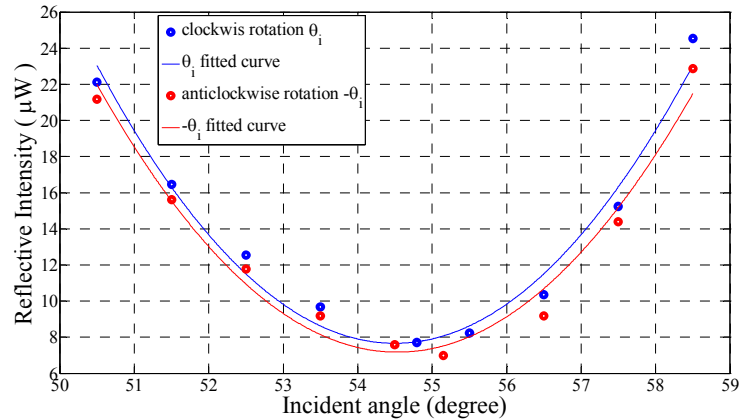

(a)

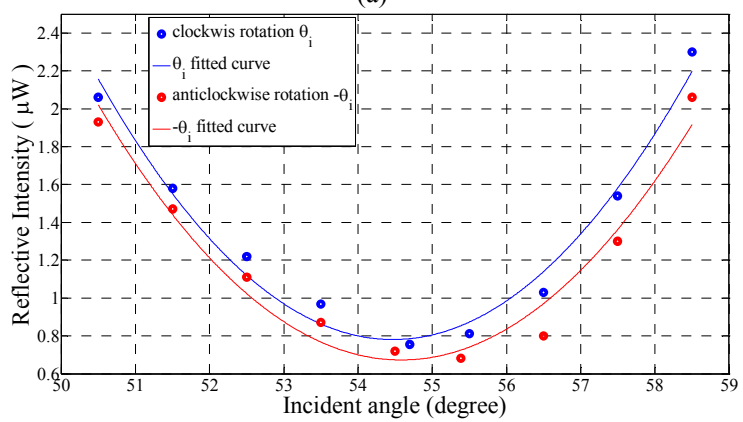

(b)

Figure 3. Measured Brewster angles on $\beta$-PVDF for two conjugate incident paths, under: (a) $100 \%$ laser intensity, (b) $10 \%$ laser intensity (only one-tenth of data points are shown)

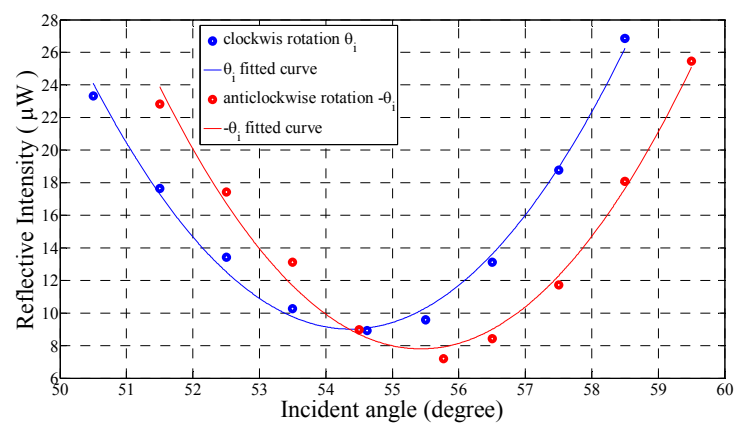

(a)

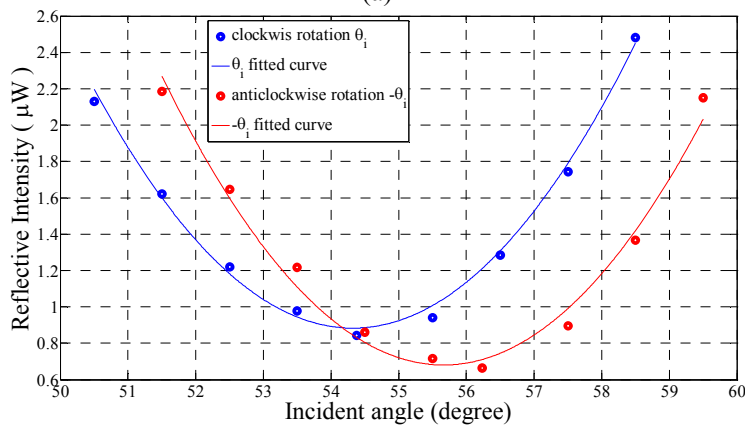

(b)

Figure 4. Measured Brewster angles on poled $\beta$-PVDF for two conjugate incident paths, under: (a) $100 \%$ laser intensity, (b) $10 \%$ laser intensity (only one-tenth of data points are shown). 
and in particular, when the laser beam is attenuated to $10 \%$, as predicted by the theory of the authors [3]. The typical data are given in Table 1.

It is noted that although even the intrinsic $\alpha$ phase PVDF possesses birefringence and this can lead to different Brewster angles as in the above too, the difference degree is at most around $0.129^{\circ}$, and hence may be ignored.

This can be verified by putting into (4) the known ordinary and extraordinary refractive indices of PVDF and getting the Brewster angles of $54.814^{\circ}$ and $54.933^{\circ}$, respectively. Further, the fact that the larger deviation is evidenced in the poled- $\beta$ phase, as compared with that from the $\beta$ phase, indicates that permanent dipoles are indeed the cause of such alteration in Brewster angles.

\subsection{Effective Permanent Polarization}

By putting the above experimental data (i.e., Table 1) into (4), the relative dielectric coefficients $\left(\tilde{\varepsilon}_{r t}\right)$ for both the $\beta$ - and poled- $\beta$ PVDF films are extracted and tabulated in Table 2.

Then, the averaged effective permanent polarization $P_{0}(\omega)$ and orientation $\theta_{0}$ can be extracted through trial-and-error (see, Table 3 ) by putting these coefficients into (2) and (3), and using the relations: $E_{t}^{p}=t_{p} \cdot E_{i}^{p}$ and $t^{p}=2 n_{\text {air }} \cos \theta_{i} /\left(n_{\text {air }} \cos \theta_{i}+n_{t} \cos \theta_{i}\right)$. In the above, $E_{i}^{p}=\sqrt{S / \varepsilon_{0} C}, \theta_{t}=\sin ^{-1}\left(n_{\text {air }} / n_{t} \sin \theta_{i}\right)$, with $S, \varepsilon_{0}$, and $C$ being the irradiating light intensity per unit area, the vacuum permittivity (i.e., $8.85 \cdot 10^{-12} \mathrm{~F} / \mathrm{m}$ ), and the light speed in vacuum, respectively, and $\varepsilon_{r}=n_{o}^{2}$.

It can be seen that the electro-poling has caused the permanent polarization to increase somewhat, and most of all, its orientation with respect to the interface to add around $20^{\circ}$.

Table 1. Brewster angles measurement.

\begin{tabular}{ccccc}
\hline Parameters & $\begin{array}{c}\text { Beam } \\
\text { intensity }\end{array}$ & $\theta_{B}\left(\theta_{i}\right)$ & $\theta_{B}\left(-\theta_{i}\right)$ & $\Delta \theta_{B}$ \\
\hline \multirow{2}{*}{$\beta$-PVDF } & $100 \%$ & $54.7925^{\circ}$ & $55.1475^{\circ}$ & $0.355^{\circ}$ \\
Poled- $\beta$ & $10 \%$ & $54.695^{\circ}$ & $55.3875^{\circ}$ & $0.6925^{\circ}$ \\
PVDF & $100 \%$ & $54.62^{\circ}$ & $55.77^{\circ}$ & $1.05^{\circ}$ \\
\hline
\end{tabular}

Table 2. Relative dielectric coefficients through fitting experimental data.

\begin{tabular}{cccc}
\hline Parameters & $\begin{array}{c}\text { Beam } \\
\text { intensity }\end{array}$ & $\widetilde{\mathcal{\varepsilon}}_{r}\left(\theta_{i}\right)$ & $\widetilde{\mathcal{\varepsilon}}_{r}\left(-\theta_{i}\right)$ \\
\hline \multirow{2}{*}{$\beta$-PVDF } & $100 \%$ & 2.008 & 1.994 \\
& $10 \%$ & 2.062 & 2.1 \\
Poled- $\beta$ & $100 \%$ & 1.983 & 1.948 \\
PVDF & $10 \%$ & 2.16 & 2.239 \\
\hline
\end{tabular}

\subsection{Novel 2D Refractive Index Ellipse}

Owing to its intrinsic uniaxial birefringence property $[5,6]$, when a light is incident upon a PVDF film (as formed, without poling), the refracted light is decomposed into an ordinary wave and an extraordinary wave, which correspond to refractive indices of $n_{o}$ and $n_{e}$, respectively. Namely, when the plane-of-incident is formed by the light's propagating direction vector $\vec{k}$ and the uniaxis $\hat{z}$ (see, Figure 5), with the angle between them being $\theta$, then a slice on the $3 \mathrm{D}$ refractive index ellipsoid cutting perpendicular to $\vec{k}$ will give rise to an elliptic contour which is of the minor axis $n_{o}$ and major axis $n_{s}$ in a relationship expressed as:

$$
\frac{1}{n_{s}^{2}(\theta)}=\frac{\cos ^{2} \theta}{n_{o}^{2}}+\frac{\sin ^{2} \theta}{n_{e}^{2}}
$$

However, the whole picture will change considerably in the presence of ordered permanent dipoles. Namely, unlike the traditional elliptic contour in red color in the polar diagram Figure 6, unconventional contours in blue and green represent $2 \mathrm{D}$ (two dimension) refractive index surfaces of the situation on $\beta$-PVDF (i.e., $\theta_{0}=41.64^{\circ}$ ) under $100 \%$ and $10 \%$ laser power, respectively; and those in purple and orange colors are on poled- $\beta$ PVDF (i.e., $\theta_{0}=61.62^{\circ}$ ) under $100 \%$ and $10 \%$ laser power, respectively. Note that, in Figure 6, as the ordinate dimension is along the direction of interface (in green) and the abscissa along the norm in real setup, both the I and III quadrants describe the refraction in the incident angle range of $0^{\circ} \sim 90^{\circ}$ (i.e., $\theta_{i}$ ), and quadrants II and IV depicts that of $0^{\circ} \sim-90^{\circ}$ (i.e., $-\theta_{i}$ ). Hence, the dipole-engineered ones would demonstrate open splittings near the traditional incident angles. Among them, the deviation should be more outstanding for the case with the test film being poled- $\beta$ than $\beta-\mathrm{PVDF}$, and especially when at lower incident laser power.

Experimentally, the following tendency was observed when the test samples were altered from normal to $\beta$ - and then to poled- $\beta$ PVDF films. When the incident angles

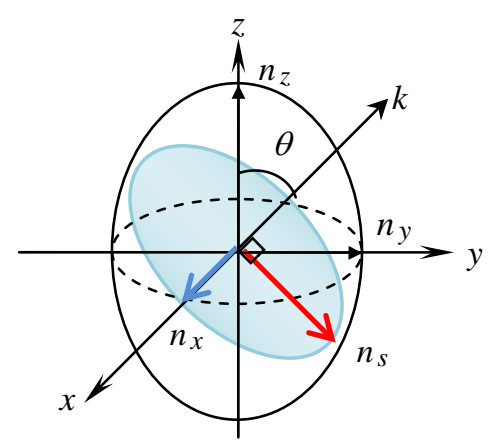

Figure 5. Construction of the refractive index surface. 


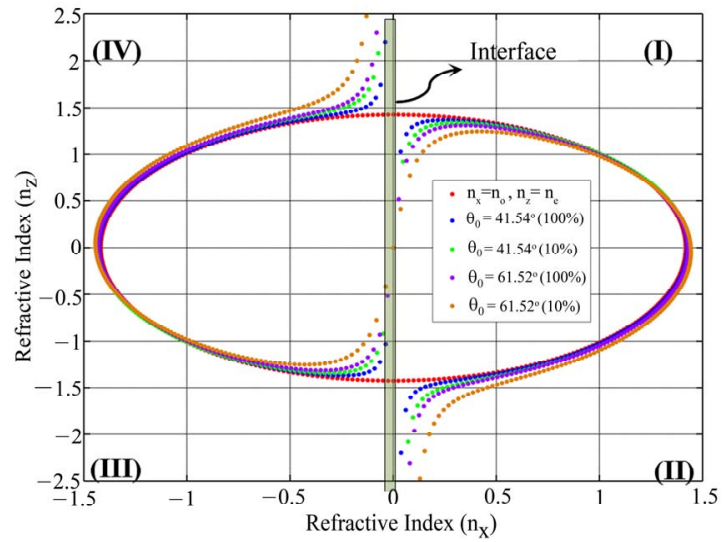

Figure 6. The polar diagram for refractive index distribution.

was within the I- and III- quadrants (i.e., $\theta_{i}$ ), the evidenced Brewster angles shrunk and the refractive index became smaller (i.e., $\tilde{\varepsilon}_{r}<\varepsilon_{r}$ ). On the other hand, when the laser was incident in the II- and IV-quadrants (i.e., $\left.-\theta_{i}\right)$, both the Brewster angles and the refractive index $\left(\tilde{\varepsilon}_{r}>\varepsilon_{r}\right)$ switched to larger values. This tendency apparently goes with the above theoretical prediction.

\subsection{Notable Indication on Traditional SPR Measurements}

Facilitated by its very high-Q resonance angle, the surface plasmon resonance (SPR) type of techniques, and their variations, are known to be very sensitive tools for measurements of refractive indices $\left(n=\sqrt{\varepsilon_{r} \mu_{r}}\right)$. Among them, the one using prism coupling and in the so-called "Kretschmann-Raether (KR) configuration" [9,10], is probably the most widely adopted practice. That is, the KR-configured materials from top down is arranged to be: prism (the " 0 " matter), metal film (the " 1 " matter), air gap, target under test (the " 2 " matter), and buffer layer (the " 3 " matter). When a p-wave is incident at a so-called "resonance angle" onto the topmost KR-configured plane (i.e., at "0"-" 1 " interface), a surface resonant plasma wave is excited at the metal-air interface, leading to a minimum in light reflection $[9,10]$.

The overall reflection coefficient (of an incident p-wave) off this 4-material KR configuration can be derived from using the Fabry-Perot interference principle and is $[9,10]$ :

$$
r_{0123}^{p}=\frac{r_{01}^{p}+\frac{r_{12}^{p}+r_{23}^{p} e^{i 2 \varphi_{2}}}{1+r_{12}^{p} r_{23}^{p} e^{i 2 \varphi_{2}}} e^{i 2 \varphi 1}}{1+r_{01}^{p} \frac{r_{12}^{p}+r_{23}^{p} e^{i 2 \varphi_{2}}}{1+r_{12}^{p} r_{23}^{p} e^{i 2 \varphi_{2}}} e^{i 2 \varphi_{2}}} e^{i 2 \varphi_{1}}
$$

And, the overall reflectivity in power is : $R=\left|r_{0123}^{p}\right|^{2}$, where $r_{01}^{p}, r_{12}^{p}, r_{23}^{p}$ are reflection coefficients at " 0 "-“1", "1"-“"2", and "2"-“3" interfaces according to the traditional Fresnel equations; and $\varphi_{i}=k_{0} \sqrt{\varepsilon_{i}} \cos \theta_{i} d_{i}$ are phase angles associated with matters " 1 " and " 2 ", respectively; and $k_{0}$ is incident wave vector; and $\varepsilon_{1} / d_{1}$ and $\varepsilon_{2} / d_{2}$ are relative dielectric coefficient / layer thickness of the metal film and material under test, respectively.

However, it is found in the above experiments that in the presence of permanent dipoles, not only is the Brewster angle dependent on the incident light power as well as the dipole orientation, but also that two conjugate incident light paths result in distinctively different refractions. Therefore, although the form of (6) remains the same in the presence of permanent dip +-+++++ oles, values of local reflection coefficients involved can vary considerably from those of their classical counterparts. In other words, the traditional confidence in SPR type of measurements may be in jeopardy when the material under test is embedded with permanent dipoles, as will be shown in what follows.

Consider a KR-configured SPR measurement setup as an example. It includes: a lens (SF 11) of relative dielectric coefficient of $(1.7786)^{2}$, a silver metal film of 52 $\mathrm{nm}\left(d_{1}\right)$ thickness of a relative dielectric coefficient of $-17.6+0.67 i$ [9], a PVDF film (as grown, or $\beta$, or poled- $\beta$ ) as the material under test of thickness of about $15 \mu \mathrm{m}\left(d_{2}\right)$, with its original relative dielectric coefficients being $d_{2}$ and $n_{e}^{2}$, and a buffer layer of air of a relative dielectric coefficient of about 1 . This configuration is then subjected to the irradiation of a light beam , from a $632.8 \mathrm{~nm}$ wavelength He-Ne laser, of the incident angles ranging within $50^{\circ} \sim 70^{\circ}\left(\theta_{i}\right)$ and its conjugate counterpart paths within the angle range $-50^{\circ} \sim$ $-70^{\circ}\left(-\theta_{i}\right)$.

The numerical calculation result based on the above setup is shown in Figure 7 and indicates the following. The birefringence (i.e., $n_{e}$ besides $n_{o}$ ) of as-grown PVDF suffices to give a maxial SPR resonance angle deviation of about $0.5^{\circ}$ (away from $58^{\circ}$ ). This deviation of resonance angles is considerably amplified in the $\beta$ $\left(2.5^{\circ}\right)$ and poled- $\beta\left(4^{\circ}\right)$ cases, owing to the increase of permanent polarization density and alignment. In such sensitive SPR type of measurements, these large deviations of resonance angles represent large distortions in the light reflectivity, as illustrated in Figure 7. Notably, it also affirms the theoretical prediction (see, (3)) that the reflectivity coefficient is inversely proportional to the strength of the incident (or, transmitted) light electric field. All these findings indicate that traditional SPR type 

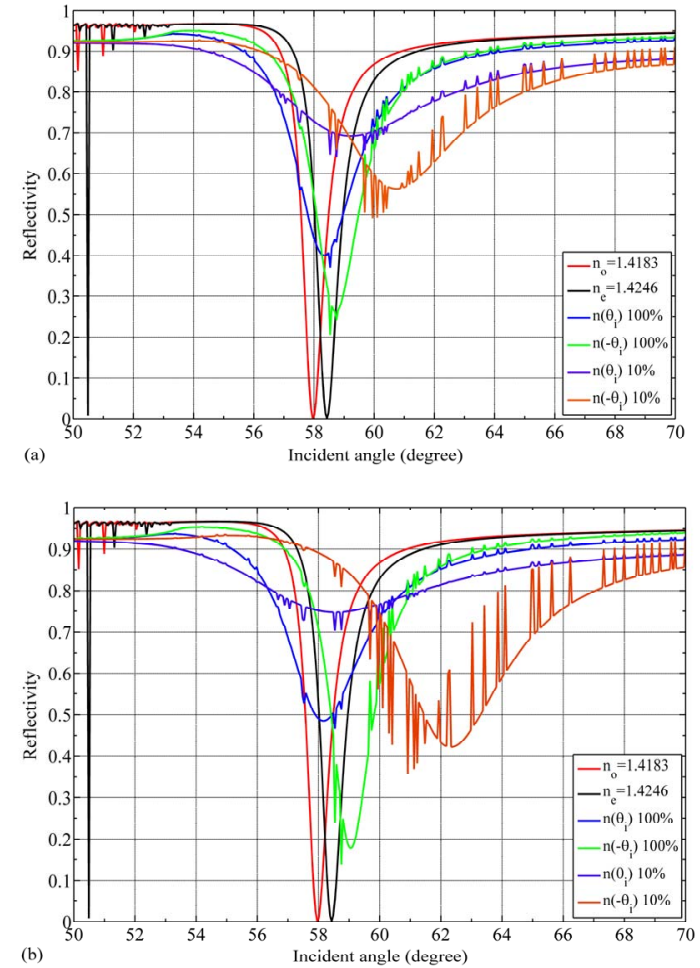

Figure 7. Calculated SPR reflectivities for: (a) $\beta$-PVDF, (b) poled- $\beta$-PVDF.

of measurements needs to exercise precaution when the material under test is embedded with permanent dipoles. For example, most living cells are with cell walls made of two opposite double layers of dipolar molecules [11].

\section{Summary and Conclusions}

It was speculated that by way of incorporating permanent dipoles within a chosen host material, the latter's Brewster angle can be altered. Experiments on PVDF films subjected to different degrees of dipole engineering not only verified the above theoretical prediction, but, when reversely extracting the effective permanent polarization density and orientation angle, also quantitatively evidenced the open splitting characteristics of the related 2D refractive index ellipse near the traditionally somewhat large incident angle. This finding apparently poses a severe challenge to the correctness of traditional SPR type of measurements when in the presence of permanent dipoles of any form.

\section{REFERENCES}

[1] E. Hecht, "Optics," 4th Edition. Addison-Wesley, New York, 2002.

[2] W. T. Doyle, "Scattering approach to Fresnel's equations and Brewster law," American Journal of Physics, Vol. 53, Issue 5, 1985, pp. 463-468.

[3] Chungpin Liao, Hsien-Ming Chang, and Chien-Jung Liao, "Manipulating the Brewster angles by using microscopically coherent dipole quanta and its possible implications," IEEE Journal Lightwave Technology, Vol. 24, No. 8, Aug 2006, pp. 3248-3254.

[4] H. A. Haus and J. R. Melcher, "Electromagnetic Fields and Energy," Prentice-Hall, New York, 1989.

[5] K. M. Yassien, M. Agour, C. von Kopylow and H.M. EI-Dessouky, "On the digital holographic interferometry of fibrous material, I: Optical properties of polymer and optical fibers," Optics and Lasers in Engineering , Vol. 48, Issue 5, 2010, pp. 555-560.

[6] M. Matsukawa, K. Shintani, S. Tomohiro and N. Ohtori, "Application of Brillouin scattering to the local anisotropy and birefringence measurements of thin layers," U1trasonics, Vol. 44, Suppl. 1, 2006, pp. e1555-e1559.

[7] R. Belouadah, D. Kendil, E. Bousbiat, D. Guyomar and B. Guiffard, "The electronic structures and magnetic properties of the vanadates M(pyrazine)V4O10 [I, M=Co; II, $\mathrm{M}=\mathrm{Zn}]$ studied by first-principles calculations," Physica B: Physics of Condensed Matter, Vol. 404, 2009, pp.1746-1751.

[8] T. T. Wang and J. E. West, "Polarization of poly (vinylidene fluoride) by application of breakdown fields," Journal of Applied Physics , Vol. 53, Issue 10, October 1982, pp. 6552-6556.

[9] H. Raether, "Surface Plasmons on Smooth and Rough Surfaces and on Gratings," Springer Tracts in Modern Physics, Springer, Berlin, 1988.

[10] S. A. Maier, "Plasmonics: Fundamental and Applications," Springer, New York, 2007.

[11] B. Alberts, A. Johnson, J. Lewis, M. Raff, K. Roberts, "Molecular Biology of the Cell," Garland Science, New York, 2007. 